JGG 2021;69:87-97

doi: $10.36150 / 2499-6564-446$

\title{
Brief intensive observation areas in the management of acute heart failure in elderly patients leading to high stabilisation rate and less admissions
}

\author{
Gabriele Savioli'1,2, Iride Francesca Ceresa ${ }^{1}$, Elena Novara², \\ Tonia Persiano ${ }^{2}$, Francesca Grulli², Giovanni Ricevuti ${ }^{3}$, \\ Maria Antonietta Bressan ${ }^{4}$, Enrico Oddone ${ }^{5}$ \\ ${ }^{1}$ Emergency Department, Irccs Policlinico San Matteo, Pavia, Italy; ${ }^{2}$ PhD University of Pavia, \\ Italy; ${ }^{3}$ Former Professor of Geriatric and Emergency Medicine, University of Pavia, Italy; ${ }^{4}$ Past \\ Director Emergency Department, Irccs Policlinico San Matteo, Pavia, Italy; ${ }^{5}$ University Researcher, \\ Dipartimento di Sanità Pubblica, Medicina Sperimentale e Forense, University of Pavia, Italy
}

Objectives. Acute heart failure is major cause of hospitalisation in Western countries. As patients with acute heart failure cannot be admitted directly to the wards, they stay in emergency rooms, causing access block. Brief Intensive Observation areas are holding units dedicated to the stabilisation of patients requiring close monitoring. However, these units have been associated with acute exacerbation of heart failure. This study aimed to evaluate the impact of Brief Intensive Observation areas on the management of acute heart failure in elderly patients.

Methods. This retrospective, single-centred observational study analysed patients who presented in the emergency room with acute heart failure in 2017 and divided them into two cohorts: those treated in the Brief Intensive Observation and those who were not. The reduction of colour codes at discharge, mortality rate within the emergency rooms, hospitalisation rate, rate of transfer to less intensive facilities and readmission rate at 7,14 and 30 days after discharge were compared.

Results. Of the 694 patients, $62 \%$ were transferred to the Brief Intensive Observation for stabilisation. Age and sex between the cohorts were not significantly different. However, compared to non-Brief Intensive Observation patients, the Brief Intensive Observation patients had worse clinical conditions on arrival and longer stabilisation period. The stabilisation rate was higher in Brief Intensive Observation patients than in non-Brief Intensive Observation patients.

Conclusions. Brief Intensive Observation areas allows effective stabilisation of elderly patients, better management of beds, reduced admission rates and reduced use of high intensity care unit beds.

Key words: brief intensive observation, acute heart failure, emergency room, holding area, decision area, elderly patients

This is an open access article distributed in accordance with the CC-BY-NC-ND (Creative Commons Attribution-NonCommercial-NoDerivatives 4.0 International) license. The article can be used by giving appropriate credit and mentioning the license, but only for non-commercial purposes and only in the original version. For further information: https://creativecommons.org/licenses/by-nc-nd/4.0/deed.en

\section{INTRODUCTION}

Acute heart failure (AHF) is a major cause of hospitalisation in Western countries, accounting for approximately $>10 \%$ of visits to the Emergency Department (ED) by patients aged over 70 years. Approximately $80 \%$ of ED patients with AHF have clinical indications for hospitalisation ${ }^{1-2}$. AHF 
accounts for $5 \%$ of all acute causes of hospitalisation and $10 \%$ of hospitalised patients and for approximately $2 \%$ of health expenditure, mainly due to hospitalisation costs. It is estimated that there will be a total mortality rate of $50 \%$ at 4 years. Among patients with AHF, mortality and re-hospitalisation are $40 \%$ per year. International databases show that, in the last decade, elderly patients (average age, 75 years) were most prone to AHF, with no significant difference between men and women ${ }^{3-5}$.

Usually, patients with AHF cannot be admitted directly to the wards. Optimal organisation of the emergency room (ER) is therefore essential to effectively manage AHF and other acute pathologies and fragile groups such as the elderly, who stay in the ER for a few days while waiting for admission. Older people often have complex home therapies including drug medications. Hospitalisation can also worsen their cognitive state, especially in ER with inadequate equipment. Holding areas are created to resolve 'access block' and 'boarding'. Access block refers to the delay in patients gaining access to inpatient beds after being admitted ${ }^{6-10}$. Numerous studies from the United States, United Kingdom, Canada and Australia have shown that access block causes ED overcrowding and affects the quality of care. 'Boarding' in ED refers to when patients awaiting inpatient beds after finishing their process in the ER ${ }^{10-16}$. Holding units are clinical decision units or observation units within the ED. In the United States, reviews by the Institute of Medicine Committee found that such units were able to alleviate access block and ED overcrowding, reduce hospitalisation and improve ambulatory care ${ }^{17,19}$. Observation units have been associated with specific clinical conditions, such as acute exacerbation of heart failure, which is a very common cause for hospital admission ${ }^{20-28}$. In contrast, some studies observed only minor improvements in using decision units: reduced length of stay (LOS) in ED, reduced admission rate and no increase in the rate of revisitation to the ED ${ }^{29-37}$. Despite these benefits, carefully planned clinical management protocols should be also in place with adequate support staff ${ }^{29-36}$.

In our Error ED reports AHF as a prevalent cause for patient visits and admission, thereby causing access block. Thus, a decision unit was formed comprising a team of experienced physicians dedicated to the holding area called Brief Intensive Observation (OBI).

This study aimed to investigate the association of $\mathrm{OBI}$ admission with a significantly high rate of patient stabilisation, a low transfer rate to other hospital wards or departments and a low incidence of For this reason we analyse: hospitalisations. We compared the reduction of colour codes at discharge, mortality rate within the $\mathrm{ED}$, hospitalisation rate, transfer rate to less intensive facilities and readmission rate at 7,14 and 30 days after discharge between $\mathrm{OBI}$ and non-OBI patients.

\section{METHODS}

\section{OVERALL DESIGN}

This study included elderly patients ( $\geq 75$ years of age) who visited the ED of San Matteo Hospital Foundation, Pavia, Italy, for AHF between January 1 and December 31,2017 . The inclusion criteria were as follows: state of consciousness not altered, ability to read and consent to the processing of data for health and research purposes. Patients were assigned to the $\mathrm{OBI}$ group or the Non-OBI group through a clinical evaluation; those who were in a worse clinical condition were assigned to the first group.

\section{STUDY DESIGN}

This retrospective single-centre observational study analysed data collected provided directly by San Matteo Hospital Foundation through the software PiEsse. Our primary outcome was the effect of the management in the $\mathrm{OBI}$ on the clinical improvement at discharge (any change in priority code such as red to green, red to yellow and yellow to green) denoting a suitable proxy for the degree of patient stabilisation. The secondary outcomes were mortality rate within the $\mathrm{ED}$, hospitalisation rate, rate of transfer to less intensive care facilities and readmission rate at 7,14 and 30 days after discharge. An ad hoc query was performed to obtain the data of interest. The name and surname of patients were substituted with an anonymous code which ensured that researchers were blind to the patient identities.

At the time of admission to the ER of San Matteo Hospital Foundation, the patients provided informed consent for the processing of data for medical and research purposes.

\section{Statistical ANALYSIS}

Statistical analyses were carried out using appropriate logistic, univariate and multivariate regression models to test the association between the assignment to the $\mathrm{OBI}$ group and clinical stabilisation (reduction of colour codes at discharge). Continuous variables were described as mean and standard deviation, while qualitative variables were expressed with counts and percentages.

Comparisons between the groups of continuous variables were made with the Student t-test, while associations between qualitative variables were studied with the $\chi 2$ test or Fisher's exact test when the number of observations within at least a single cell was equal to or lower than 5.

The significance level has been set at alpha 0.05 (statistical significance at $p$-value $<0.05$ ) and all tests were 
two-sided. The analyses were conducted with STATA software, version 14 (Stata Corporation, College Station, 2015, Texas, USA).

\section{RESULTS}

This study enrolled 694 consecutive elderly patients (male, 322, 46\%; female, 372, 54\%; mean age, 85 years) presenting with AHF who visited the ED of San Matteo Hospital Foundation. The presence of arrhythmia, heart rate $(H R)$, systolic and diastolic blood pressure (SBP, DBP), arterial oxygen saturation $\left(\mathrm{SatO}_{2}\right)$, priority code at access, priority code at discharge, wait time, process time and LOS were not significantly different between men and women.

Of these patients, a total of $431(62.10 \%)$ were included in the OBI group, while 263 (37.89\%) were in the Non-OBI group. The main features of the two groups are reported in Table I. The vital signs were not significantly different between the groups, except for a higher mean HR for male patients in the OBI group. While the distribution of sex or age in the two groups was not significantly different, the male patients in the $\mathrm{OBI}$ group showed a significantly higher mean age than in the Non-OBI group. Patients in the OBI group had

Table I. Principal clinical and process features of $\mathrm{OBI}$ and Non-OBI groups.

\begin{tabular}{|c|c|c|c|c|c|}
\hline & $\begin{array}{c}\text { OBI } \\
\text { n (\%) }\end{array}$ & $\begin{array}{c}\text { Mean } \\
\text { (95\% IC) }\end{array}$ & $\begin{array}{c}\text { NON-OBI } \\
\text { n (\%) }\end{array}$ & $\begin{array}{c}\text { Mean } \\
(95 \% \text { IC) }\end{array}$ & $\mathbf{P}$ \\
\hline \multicolumn{6}{|l|}{ Sex } \\
\hline Men & $203(47.1 \%)$ & - & $119(45.2 \%)$ & - & \\
\hline Women & $228(52.9 \%)$ & - & $144(54.8 \%)$ & - & 0.635 \\
\hline \multicolumn{6}{|c|}{ Age (years) } \\
\hline Men & $203(47.1 \%)$ & $84.6(78.0-80.4)$ & $119(45.2 \%)$ & $84.2(83.4-85.0)$ & 0.549 \\
\hline Women & $228(52.9 \%)$ & 85.6 (84.9-86.3) & $144(54.8 \%)$ & $82.2(80.8-83.5)$ & 0.719 \\
\hline All & $431(100 \%)$ & $85.1(84.6-85.6)$ & $263(100 \%)$ & $85.0(84.4-85.7)$ & 0.860 \\
\hline \multicolumn{6}{|c|}{ Arrhythmia } \\
\hline Yes & $27(6.3 \%)$ & - & $13(4.9 \%)$ & - & \\
\hline No & 404 (93.7\%) & - & $250(95.1 \%)$ & - & \\
\hline All & $431(100 \%)$ & - & $263(100 \%)$ & - & 0.469 \\
\hline \multicolumn{6}{|c|}{ HR (bpm) } \\
\hline Men & $203(47.1 \%)$ & $86.4(83.7-89.0)$ & $119(45.2 \%)$ & $81.0(77.8-84.2)$ & 0.013 \\
\hline Women & $228(52.9 \%)$ & 88.0 (84.8-91.3) & $144(54.8 \%)$ & 88.1 (84.4-91.8) & 0.971 \\
\hline All & $431(100 \%)$ & $87.3(85.1-89.4)$ & $263(100 \%)$ & $84.9(82.4-87.4)$ & 0.174 \\
\hline \multicolumn{6}{|c|}{ SBP $(\mathrm{mmHg})$} \\
\hline Men & $203(47.1 \%)$ & $140.7(132.7-142.5)$ & $119(45.2 \%)$ & 137.6 (132.7-142.5) & 0.324 \\
\hline Women & $228(52.9 \%)$ & 143.9 (140.5-147.3) & $144(54.8 \%)$ & 140.1 (135.8-144.) & 0.174 \\
\hline All & $431(100 \%)$ & 142.4 (139.9-144.9) & $263(100 \%)$ & 138.9 (135.7-142.2) & 0.102 \\
\hline \multicolumn{6}{|c|}{ SBP > $180 \mathrm{mmHg}$} \\
\hline Men & $12(5.9 \%)$ & 197.8 (186.6-208.9) & $6(5.0 \%)$ & $195.7(180.0-209.3)$ & 0.713 \\
\hline Women & $17(7.5 \%)$ & 195.1 (190.6-199.6) & $7(4.9 \%)$ & $197.9(187.8-208.0)$ & 0.879 \\
\hline All & $29(6.7 \%)$ & $196.2(191.3-201.1)$ & $13(4.9 \%)$ & 194.6 (188.7-200.5) & 0.694 \\
\hline \multicolumn{6}{|c|}{ DBP (mmHg) } \\
\hline Men & $203(47.1 \%)$ & $78.7(76.4-80-9)$ & $119(45.2 \%)$ & $78.8(75.9-81.7)$ & 0.933 \\
\hline Women & $228(52.9 \%)$ & $79.3(77.0-81.7)$ & $144(54.8 \%)$ & 75.9 (73.1-78.7) & 0.065 \\
\hline All & $431(100 \%)$ & $79.0(77.4-80.6)$ & $263(100 \%)$ & 77.2 (75.2-79.2) & 0.170 \\
\hline \multicolumn{6}{|c|}{ DBP > $110 \mathrm{mmHg}$} \\
\hline Men & $4(2.0 \%)$ & 125.0 (103.5-146.5) & $3(2.5 \%)$ & $123.3(109.0-137.7)$ & 0.852 \\
\hline Women & $9(3.9 \%)$ & $119.7(116.2-123.2)$ & $2(1.4 \%)$ & $115.5(58.3-172.7)$ & 0.293 \\
\hline All & $13(3.0 \%)$ & $121.3(116.4-126.2)$ & $5(1.9 \%)$ & $120.2(111.9-128.5)$ & 0.791 \\
\hline \multicolumn{6}{|l|}{$\mathrm{SatO}_{2}$} \\
\hline Men & $203(47.1 \%)$ & $94.5(93.8-95.2)$ & $119(45.2 \%)$ & $94.7(93.8-95.7)$ & 0.657 \\
\hline Women & $228(52.9 \%)$ & $93.6(92.8-94.4)$ & $144(54.8 \%)$ & $93.8(92.7-94.9)$ & 0.771 \\
\hline All & $431(100 \%)$ & $94.0(93.5-94.6)$ & $263(100 \%)$ & $94.2(93.5-95.0)$ & 0.663 \\
\hline
\end{tabular}




\begin{tabular}{|c|c|c|c|c|c|}
\hline & $\begin{array}{c}\text { OBI } \\
\text { n (\%) }\end{array}$ & $\begin{array}{c}\text { Mean } \\
(\mathbf{9 5 \%} \text { IC) }\end{array}$ & $\begin{array}{c}\text { NON-OBI } \\
\text { n (\%) }\end{array}$ & $\begin{array}{c}\text { Mean } \\
(95 \% \text { IC) }\end{array}$ & $\mathbf{P}$ \\
\hline \multicolumn{6}{|c|}{ SatO $_{2}<85 \%$} \\
\hline Men & $9(4.4 \%)$ & $80.0(74.1-85.9)$ & $3(2.5 \%)$ & $76.0(50.1-101.9)$ & 0.485 \\
\hline Women & $18(7.9 \%)$ & $77.9(75.0-80.8)$ & $9(7.6 \%)$ & $75.8(70.2-81.4)$ & 0.421 \\
\hline All & $27(6.3 \%)$ & $78.6(76.1-81.1))$ & $12(4.6 \%)$ & $75.8(71.0-80.7)$ & 0.249 \\
\hline \multicolumn{6}{|c|}{ Priority Code - access } \\
\hline Green & $83(19.3 \%)$ & - & $73(27.8 \%)$ & - & \\
\hline Yellow & $303(70.3 \%)$ & - & 156 (59.3\%) & - & \\
\hline Red & $45(10.4 \%)$ & - & $34(12.9 \%)$ & - & 0.010 \\
\hline \multicolumn{6}{|c|}{ Priority Code - discharge } \\
\hline Green & $178(41.4 \%)$ & - & $90(35.2 \%)$ & - & \\
\hline Yellow & $246(57.2 \%)$ & - & $155(60.5 \%)$ & - & \\
\hline Red & $6(1.4 \%)$ & - & $11(4.3 \%)$ & - & 0.025 \\
\hline \multicolumn{6}{|c|}{ Wait time (min) } \\
\hline Men & $203(47.1 \%)$ & $50.6(43.8-57.3)$ & $119(45.2 \%)$ & $59.4(47.2-71.6)$ & 0.397 \\
\hline Women & $228(52.9 \%)$ & $50.1(42.9-57.3)$ & $144(54.8 \%)$ & $58.3(47.8-68.9)$ & 0.187 \\
\hline All & $431(100 \%)$ & $51.7(46.4-56.9)$ & $263(100 \%)$ & $58.8(50.9-66.8)$ & 0.126 \\
\hline \multicolumn{6}{|c|}{ Process time (min) } \\
\hline Men & $203(47.1 \%)$ & $580.9(527.4-634.5)$ & $119(45.2 \%)$ & $319.9(272.3-367.5)$ & $<0.001$ \\
\hline Women & $228(52.9 \%)$ & $652.0(599.9-704.1)$ & $144(54.8 \%)$ & $355.1(303.9-406.3)$ & $<0.001$ \\
\hline All & $431(100 \%)$ & $618.5(581.2-655.9)$ & $263(100 \%)$ & $339.2(304.0-374.4)$ & $<0.001$ \\
\hline \multicolumn{6}{|c|}{ Total time (min) } \\
\hline Men & $203(47.1 \%)$ & $624.1(566.5-681.7)$ & $119(45.2 \%)$ & $367.6(322.3-412.9)$ & $<0.001$ \\
\hline Women & $228(52.9 \%)$ & $670.3(614.5-726.1)$ & $144(54.8 \%)$ & $407.5(356.4-458.6)$ & $<0.001$ \\
\hline All & $431(100 \%)$ & $648.6(608.6-688.5)$ & $263(100 \%)$ & $389.5(354.9-424.0)$ & $<0.001$ \\
\hline
\end{tabular}

worse clinical conditions on arrival, as indicated by a significantly higher percentage of 'yellow' and 'red' codes ( $p=0.010)$, and by contrast, a better clinical status at discharge with a contemporary higher rate of green codes and lower percentage of 'red' and 'yellow' codes, compared to the Non-OBI group ( $p=0.025)$.

Table II presents the primary outcomes between the groups. The effect of the management in the $\mathrm{OBI}$ on clinical improvement seems to be more evident in patients coded with yellow code at admission. The best stabilisation in the $\mathrm{OBI}$ was statistically significant as a whole $(p=0.017)$ and in the different colour codes $(p=0.037)$, as reported in Table III. Waiting time was not significantly different among patients in the $\mathrm{OBI}$ group compared to those in the Non-OBI group ( $p=0.126)$. Patients in the $\mathrm{OBI}$ group had a significantly $(\mathrm{p}=0.029)$ longer process time (mean: 618.5 min vs 339.2 min; $p<0.001$ ) and a longer LOS (648.6 min vs 389.5 min; $p<0.001$ ); LOS was the duration of stay in the $E R$, including waiting for the medical examination, the processing time and boarding. No difference in mortality rate was observed between the two groups; however, the OBI group had a significantly higher transfer rate to other hospital wards or departments and a significantly lower hospitalisation rate. This result was also confirmed when we adjusted for all potential confounding variables. No significant differences were observed regarding patients' readmission at 7, 14 and 30 days after discharge (Tab. II).

Finally, the univariate logistic regression model (Tab. IV), in which only the independent variable $\mathrm{OBI}$ (yes or no) was tested, showed that patients in the $\mathrm{OB}$ group were not classified as 'worse condition' at discharge, in terms of any change in priority code such as red to green, red to yellow, or yellow to green ( $p=0.001)$, as confirmed by a multivariate logistic regression analysis (Table IV, Fig. 1). Thus, we can argue that with an increase in risk of $0.008 \%$ per unit increase in waiting time, a higher waiting time value provides a little increase in risk and management in the $\mathrm{OBI}$ seem to play a protective role to avoid the worst outcome at discharge.

\section{DISCUSSION}

Our ER is divided into areas dedicated to specific intensities of care: low intensity and medium-high intensity. Patients who arrive at our ED are first subjected to triage where specialised nurses with advanced business 
Table II. Frequency of principal outcome by group.

\begin{tabular}{|c|c|c|c|c|c|}
\hline & \multicolumn{2}{|c|}{ OBI } & \multicolumn{2}{|c|}{ NON-OBI } & \multirow[t]{2}{*}{$\mathbf{P}$} \\
\hline & $\mathbf{N}$ & $\%$ & $\mathbf{N}$ & $\%$ & \\
\hline \multicolumn{6}{|l|}{ Death } \\
\hline Yes & 3 & $0.70 \%$ & 2 & $0.76 \%$ & \\
\hline No & 428 & $99.30 \%$ & 261 & $99.24 \%$ & 1.000 \\
\hline \multicolumn{6}{|l|}{ Hospitalization } \\
\hline Yes & 253 & $58.70 \%$ & 172 & $65.40 \%$ & \\
\hline No & 178 & $41.30 \%$ & 91 & $34.60 \%$ & 0.079 \\
\hline \multicolumn{6}{|l|}{ Transfer* } \\
\hline Yes & 70 & $16.24 \%$ & 18 & $6.84 \%$ & \\
\hline No & 361 & $83.76 \%$ & 245 & $93.16 \%$ & $<0.001$ \\
\hline \multicolumn{6}{|l|}{ Outcomes } \\
\hline Hospitalization & 253 & $58.70 \%$ & 172 & $68.44 \%$ & \\
\hline Discharge & 102 & $23.67 \%$ & 83 & $23.18 \%$ & \\
\hline Transfer* $^{*}$ & 70 & $16.24 \%$ & 23 & $6.42 \%$ & \\
\hline Voluntary leaving & 3 & $0.70 \%$ & 4 & $1.12 \%$ & \\
\hline Death & 3 & $0.70 \%$ & 2 & $0.76 \%$ & 0.004 \\
\hline \multicolumn{6}{|l|}{ Readmission } \\
\hline Yes & 50 & $11.60 \%$ & 29 & $11.03 \%$ & \\
\hline No & 381 & $88.40 \%$ & 234 & $89.97 \%$ & 0.817 \\
\hline \multicolumn{6}{|c|}{ Readmission at 7 days } \\
\hline Yes & 11 & $2.55 \%$ & 10 & $3.80 \%$ & \\
\hline No & 420 & $97.45 \%$ & 253 & $96.20 \%$ & 0.351 \\
\hline \multicolumn{6}{|c|}{ Readmission at 14 days } \\
\hline Yes & 24 & $5.57 \%$ & 19 & $7.22 \%$ & \\
\hline No & 407 & $94.43 \%$ & 244 & $92.78 \%$ & 0.380 \\
\hline \multicolumn{6}{|c|}{ Readmission at 30 days } \\
\hline Yes & 52 & $12.06 \%$ & 32 & $12.17 \%$ & \\
\hline No & 379 & $87.94 \%$ & 231 & $87.83 \%$ & 0.968 \\
\hline
\end{tabular}

${ }^{\star}$ Transfers to other hospital wards or structures

Table III. In the following we present a separate analyses by different downgraded color codes (red to yellow, red to green, yellow to green).

\begin{tabular}{|l|c|c|c|c|}
\hline \multicolumn{1}{|c|}{ Outcome } & \multicolumn{3}{|c|}{ OBI } & \\
\hline No improvement & No & Yes & Total & \\
\hline Red to yellow & $184(69.96 \%)$ & $263(61.02 \%)$ & $447(64.41 \%)$ & \\
\hline Red to green & $23(8.75 \%)$ & $34(7.89 \%)$ & $57(8.21 \%)$ & \\
\hline Yellow to green & $5(1.90 \%)$ & $8(1.86 \%)$ & $13(1.87 \%)$ & \\
\hline
\end{tabular}

training collect information related to the patient's general data, the main presenting symptoms and a short history. They then proceed to the measurement of vital signs and conduct a visual inspection. At this stage, based on 'triage grids' drawn up mainly based on the evolution of the main symptoms, the patient's medical history and vital signs, the patients are assigned a priority code for the medical examination and are directed to an area of appropriate intensity of care.
There are five levels of priority code for the medical examination in our ED:

a) Red code: immediate entry into the shock room (high intensity area). It is assigned to patients with severe impairment of vital signs or consciousness;

b) Yellow code, medium intensity: immediate, or at least within 40 minutes, entry to the average intensity care area; 
Table IV. Results of univariate and multivariate logistic regression models. Comparison of improvement at discharge (any change in priority code such as red to green, red to yellow, yellow to green) $v s$ no improvement at discharge, by selected variable.

\begin{tabular}{|l|c|c|c|}
\hline & OR & $95 \% \mathrm{CI}$ & $\boldsymbol{P}$ \\
\hline Univariate analysis & & & \\
\hline Non OBI & 1 (reference) & - & 0.001 \\
\hline OBI & 0.579 & $0.433-0.774$ & 0.001 \\
\hline Multivariate analysis & & & 0.035 \\
\hline OBI (yes vs no) & 0.583 & $0.419-0.812$ & 0.922 \\
\hline Age (year) & 0.984 & $0.970-0.998$ & 0.199 \\
\hline Sex (male vs female) & 1.015 & $0.756-1.362$ & 0.369 \\
\hline Arrhythmia (yes vs no) & 1.575 & $0.788-3.148$ & 0.032 \\
\hline HR (bmp) & 0.997 & $0.990-1.004$ & 0.898 \\
\hline Sato ${ }_{2}(\%)$ & 0.972 & $0.947-0.998$ & 0.183 \\
\hline SBP (mmHg) & 1.000 & $0.994-1.007$ & 0.001 \\
\hline DBP (mmHg) & 0.992 & $0.981-1.004$ & 0.752 \\
\hline Wait time (min) & 1.008 & $1.005-1.012$ & 0.001 \\
\hline Process time (min) & 1.000 & $0.999-1.001$ & \\
\hline Total time (min) & 1.000 & $0.999-1.001$ & \\
\hline
\end{tabular}

HR: Hearth Rate; SBP: Systolic blood pressure; DBP: Diastolic blood pressure; Sat0 ${ }_{2}$ : Oxygen saturation.

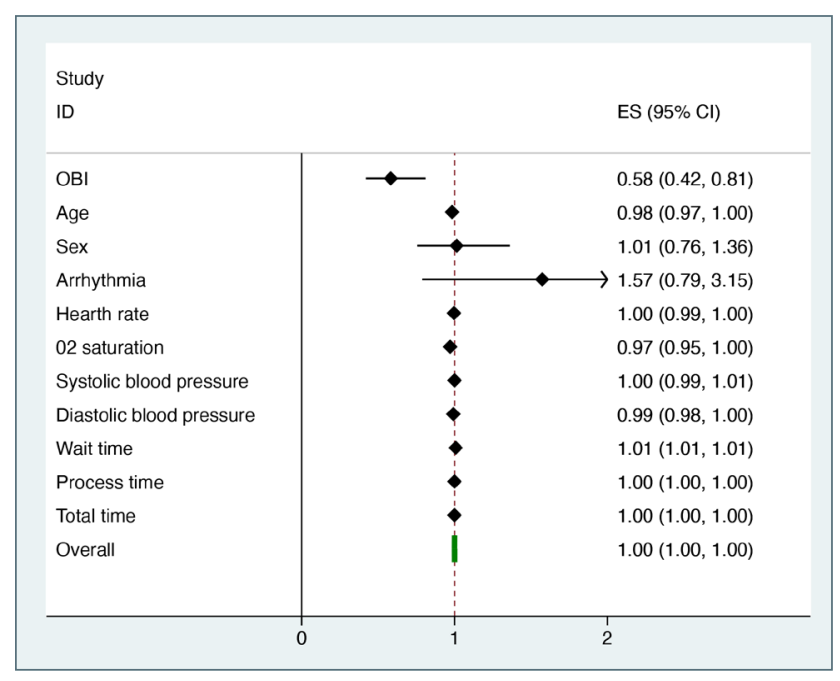

Figure 1. Forrest plot for multivariate logistic regression model results showed in Table IV.

c) Yellow code, low intensity: immediate entry, or at least within 40 minutes, to the low intensity care area;

d) Green code: assigned to deferred urgency or minor emergencies with a wait of a few hours and entry to the low intensity of care area;

e) White code: non-urgent cases with a wait of a few hours and entry to the low intensity of care area.

The criteria for assigning a patient to the medium-high intensity care area include the deterioration of a vital sign or consciousness, or the worsening of any concomitant symptoms (e.g. typical chest pain), the need for care (e.g. oxygen), or the need for multi-parameter monitoring.

The patient is then seen by the ER physician who will set the patient's therapeutic and diagnostic pathway. The two different areas of intensity of care converge on the stabilisation area, which is the OBI. The physicians in the room can use their clinical judgement to admit the patient directly without going to the OBI. At the end of the process, patients are admitted, discharged, or transferred to a hospital with a lower intensity of care depending on the degree of illness severity and the stabilisation achieved. The patient's condition on discharge or referral is categorised by the physician using a colour code. A red code is given to unstable patients, and yellow and green codes to patients who are stabilised but still in need of medium and low intensity care, respectively.

Measuring patient stabilisation and acuity presents objective difficulties and has no direct indices. We therefore chose composite indirect indices: the reduction of the codes at discharge, the mortality rate in the ED, the hospitalisation rate, the transfer rate to less intensive care hospitals and readmission rate at 7,14 and 30 days after discharge.

In the comparison between the triage code and the severity code at discharge representing the primary outcomes, the code attributed to triage expresses both a priority to medical examination and treatment and the need to receive a low, medium, or high intensity of treatment ${ }^{38-40}$. In our study, as both codes were defined based on the need for low or high intensity of care, the comparison between the codes was used to evaluate patient stabilisation. 
In more detail, the rate of downgrading of colour codes from triage to discharge represented the stabilisation outcome, thereby better highlighting the impact of the management in the $\mathrm{OBI}$ towards patients who most need stabilisation (yellow and red codes) and multiparametric monitoring of vital signs. The red (or 1 in the international CTAS and ESI systems) and yellow codes (or 2 in the international CTAS and ESI systems) have shown in fact to have more unfavourable outcomes at discharge ${ }^{41-43}$.

The diagnostic and therapeutic assistance process begins at the triage ${ }^{41-48}$. There are many triage models adopted worldwide, so they are distinguished mainly by the different number of priority visit codes. In the 1980s, the number of 3- and 4-level triages increased ${ }^{38-48}$. Gerald Fitzgerald introduced the first 5-level triage in 1986 in Australia ${ }^{49}$, which demonstrated superiority criteria especially as regards its correlation with the patient's real acuity and with inter- and intra-observer reproducibility.

In Italy, a classification according to priority levels has been used since 1990. Subsequently, the national legislation established a classification according to four levels of priority identified by the colours red, yellow, green and white ('for homogeneity, visual effectiveness and understanding also by of patients'; State-Regions Conference 2001) ${ }^{50}$.

In 2012, the Professionals and Scientific Societies gathered in the National Triage Coordination proposed a coding system with 5 priority levels using a numerical code from 1 to 5. Many other countries such as Canada, United States, United Kingdom, Australia, New Zealand, Austria, Finland, Brazil, Barbados, Japan, Spain and Saudi Arabia have been implementing a global triage method and hoping for an organised treatment algorithm based on the intensity levels of care and complexity. For this reason, our foundation, after a worldwide review of the literature on triage, have been implementing since 2015 a five-level triage introducing the 'yellow low intensity of care' code, roughly equivalent to code 3 of the CTAS and the ESI ${ }^{41-46}$.

Monitoring of the triage process has been active in our institute since 1991 with semi-annual reports and was practised throughout the entire study period.

The exit codes, on the other hand, are severity codes for discharge, based on clinical criteria were attributed to patients by their ER physicians in charge at the time. They maintain the same triage level classification (colour or number).

At the end of 2016, physicians from our hospital's ED were chosen to join the $\mathrm{OBI}$ team. The $\mathrm{OBI}$ had as its mission the safe discharge or appropriate admission of patients, and to assist with the bed management of all emergency admissions. Due to boarding and overcrowding, the need to develop an area in which to stabilise acute patients had become urgent ${ }^{10-18}$. Our hospital had no emergency medicine or stabilisation area, so the OBI team was appointed for this purpose because it was already functioning and it consisted of a small pool of physicians who had developed a closeness and homogeneity of patient management ${ }^{19,20-23,25-}$ 26. Given the wide range and complexity of patients, bed management (with vacancies arising from various departments throughout the day) in a second-level ED requires 12-hour shifts, to create a more continuous and homogeneous service. Other ED personnel render 6.5-hour shifts to avoid a deteriorating performance by the physicians. From an organisational point of view, the $\mathrm{OBI}$ team is responsible for the management of beds for acute admissions. Their clinical duties included management of the patients sent by the different intensity care areas after an initial evaluation by the attending physician or they could admit a patient directly from the waiting room in case of overcrowding. They also managed patients in boarding and they stabilised complex patients who needed an average intensity of care. They assess the functional capacity of patients, to assist in making clinical decisions and determine the need for home support for patients who are to be discharged, and differentiate high-risk patients who need hospitalisation from low-risk patients.

Patients were managed in $\mathrm{OBI}$, an area of medium intensity of care; upon entry, they underwent a reassessment as well as ECGs and laboratory tests if required and completed the diagnostic process with first- or second-level imaging if needed. Physicians will refer to each patient's therapy sheet so that the patient would continue on their existing drug therapy to avoid polytherapy. Management in a medium intensity area also allowed close multi-parameter monitoring of patients. From the outset, this proved extremely beneficial for the patient, because in an acute setting 'time is life', and this type of system combined the regular and timely application of all the treatment that the patient needed, combined with close monitoring on the same emergency platform as the ER 20-21,37.

In our experience, this management model has shortened waiting times, improved the appropriateness of admissions, optimised the use of available health resources and allowed better management of complex and serious patients that often crowd EDs allowing them to be stabilised.

\section{EVALUATION OF OUR EXPERIENCE}

The proportion of emergencies on medical examination are directed to the $\mathrm{OBI}$, while patients presenting with less urgent conditions are more often managed in the other ED areas, because patients with a greater need of 
stabilisation were sent to the dedicated area to manage the most complex patients. These data are in line with those in the international literature, as stable patients with low-risk AHF are usually managed in the ED and discharged home ${ }^{23-28}$.

Process and LOS times were much higher, as expected ${ }^{23-28}$, for patient stabilisation. Achieving stabilisation requires more processing time with longer stays in the $\mathrm{ER}$, and stabilising older patients takes longer than younger patients.

Nevertheless, this reduced hospital admission and the use of high intensive bed and increased the number of patients transferred to hospitals with lower care intensity and dimission rate. This resulted in both better management of available health resources and valuable beds and reduction of 'improper' admissions that would otherwise be managed at home. Hospitalised elderly people, who often have mild cognitive decay, experience worse outcomes ${ }^{29}$. Reducing admissions otherwise manageable at home means reducing even the worst outcomes in the elderly.

In our study mortality was not statistically significantly reduced, possibly consistent with some studies that have reported that mortality was unchanged when comparing patients who were managed in an observation unit and those who were admitted directly from the ED ${ }^{30}$. The lack of reduction in mortality in this class of patients may also be due in part to the reduced therapeutic chances in such acute events at a very advanced age. There must be end-of-life paths. Therefore, necessary care, maximum decorum and closeness of relatives must be ensured. A holding area can also meet these needs ${ }^{31-35}$.

However, the degree of stabilisation of patients achieved was significantly higher as demonstrated by the discharge code and the higher rate of transfer to hospitals with less care intensity.

Stabilisation is confirmed when the colour code downgrades from triage to discharge; it is more applicable for emergencies (yellow code or 2). This result is not surprising because patients with a lower priority code (green or white) require less stabilisation that can be done more easily even in examination rooms. The red code (or code 1), on the other hand, are often very serious cases of terminal imbalance, which require long hospitalisations and whose stabilisation cannot take place within the times of an emergency. The yellow code (or code 2) is represented by patients with impaired respiratory exchanges but who can be stabilised effectively in a shorter period in ED and be discharged or sent back to low intensity care centres.

Our findings are in line with those of some studies showing that the admission rates to an observation unit to the ED due to AHF and to both the observation unit and inpatient unit for $\mathrm{AHF}$ at 90 days reduce the rates of return ${ }^{28}$. Other studies have suggested that a specialised AHF observation unit may be best for patient care while reducing admission rates ${ }^{28-31}$. Observation units provide a cost-effective alternative compared to hospital admission for those with non-high-risk HF 35,36 by avoiding ordinary hospitalisation.

In our opinion, this may be because immediately availing the patient of the prescribed acute therapy and with the maintenance of home therapy means regaining a period of treatment that could otherwise be lost. The transfer of the patients to the duty staff may not guarantee the optimal timing of emergency therapy, or delays due to overcrowding may interrupt the normal administration of the home therapy. Furthermore, some types of drugs may not be normally stocked in the ER. Seeing the evolution and response of the patient to therapy over time allows a better stratification of the risk. The longer process time also allows the patient to be monitored with cardiac and chest ultrasound to allow a careful assessment of risk and stabilisation.

The greater degree of patient stabilisation brings the great advantage of a more marked use of beds in low intensity wards and the increased transfer to outlying hospitals with lower levels of intensity of care ${ }^{28-30,36}$. Hospitals with lower intensity levels of care have often more chances to perform physiotherapy because they can prevent immobilisation syndrome, related in the elderly with unfavourable outcomes and major complications.

This fact, associated with a physical area dedicated to the treatment of these patients, equipped with a bathroom and comfortable beds (the same as the wards), bedside tables and a hospital canteen service, in our opinion, has improved the degree of patient satisfaction as evidenced by the reduction in patients who left before being seen.

The readmission rate was lower for patients managed in OBI but was not statistically significant. This too may depend on nuance, because some studies with larger cohorts have reported an advantage in terms of returning patients. However, it should also be noted that outcomes of 30-day readmission and recurrent ED visits due to AHF or mortality remain unchanged when comparing patients managed in an observation unit and those who were hospitalised directly from the ED ${ }^{30}$.

\section{FUTURE PERSPECTIVE}

This model can be applied in situations such as ours, where there is a limited availability of medium intensity care beds in the hospital. For the best outcomes and the best management of available health resources, we propose a model in which a dedicated team, perhaps rotating, takes care of both the stabilisation of complex 
patients and their admission, together with appropriate bed management.

\section{LIMITATIONS}

First, our conclusions are limited by the observational nature of the study, including partly retrospective retrieval of information. Second, we did not compare the care patients received. Our outcomes may therefore have been affected by differening the different correctness or timeliness of the treatment. Another limitation of the study is that we do not have an echocardiographic or biochemical stratification of patients with heart failure. We therefore do not know whether the results are worth, for example, more for a heart failure with a major diastolic or sstolological component. We also point out that a true shared typing of AHF is not yet defined and that many international studies have begun to do so. However, we do not believe that this data affect the conclusions, as the advantage of this observational study is that it analyses the real life of our ER.

\section{CONCLUSIONS}

Data from our study show that a dedicated area of the $\mathrm{ER}$, such as the $\mathrm{OBI}$, with a dedicated team of doctors resulted in very good feedback on elderly patient stabilisation and has resulted in better management of beds, reduced admission rates and reduced use of high intensity care beds

\section{AUtHOR CONTRIBUTIONS}

Concept/design: Gabriele Savioli. Data analysis/interpretation: Gabriele Savioli, Iride Francesca Ceresa, Maria Antonietta Bressan, Enrico Oddone. Drafting article: Gabriele Savioli, Enrico Oddone. Critical Revision and approval: Gabriele Savioli, Iride Francesca Ceresa, Giovanni Ricevuti, Enrico Oddone, Maria Antonietta Bressan involved in Critical Revision and approval. Statistics: Enrico Oddone.

\section{References}

1 Piotr Ponikowski, Voors AA, Anker SD, et al. 2016 ESC Guidelines for the diagnosis and treatment of acute and chronic heart failure, the Task Force for the diagnosis and treatment of acute and chronic heart failure of the European Society of Cardiology (ESC), developed with the special contribution of the Heart Failure Association (HFA) of the ESC. Eur Heart J 2016;37: 2129-200. https://doi. org/10.1093/eurheartj/ehw128
2 Kenneth Dickstein, Cohen-Solal A, Filippatos G, et al. Linee guida ESC per la diagnosi e il trattamento dello scompenso cardiaco acuto e cronico 2008: Task Force per la Diagnosi e il Trattamento dello Scompenso Cardiaco Acuto e Cronico 2008 della Società Europea di Cardiologia. Linee guida elaborate in collaborazione con la Heart Failure Association dell'ESC (HFA) e approvate dalla European Society of Intensive Care Medicine (ESICM). G Ital Cardiol 2009;10:141-98.

3 Adams KF Jr, Fonarow GC, Emerman CL, et al. Characteristics and outcomes of patients hospitalized for heart failure in the United States: rationale, design, and preliminary observations from the first 100,000 cases in the Acute Decompensated Heart Failure National Registry (ADHERE). Am Heart J 2005;149:209. https://doi.org/10.1016/j. ahj.2004.08.005

4 Fonarow GC, Stough WG, Abraham WT, et al. Characteristics, treatments, and outcomes of patients with preserved systolic function hospitalized for heart failure: a report from the OPTIMIZE-HF Registry. J Am Coll Cardiol 2007;50:768. https://doi.org/10.1016/j.jacc.2007.04.064

5 Cleland JG, Swedberg K, Cohen-Solal A, et al. The Euro Heart Failure Survey of the EUROHEART survey programme. A survey on the quality of care among patients with heart failure in Europe. The Study Group on Diagnosis of the Working Group on Heart Failure of the European Society of Cardiology. The Medicines Evaluation Group Centre for Health Economics University of York. Eur J Heart Fail 2000;2:123. https://doi.org/10.1016/s0195-668x(02)00823-0

6 Moskop JC, Sklar DP, Geiderman JM, et al. Emergency department crowding, part 1 - concepts, causes, and moral consequences. Ann Emerg Med 2009;53:605-11. https://doi.org/10.1016/j.annemergmed.2008.09.024

7 Moskop JC, Sklar DP, Geiderman JM, et al. Emergency department crowding, part 2 - barriers to reform and strategies to overcome them. Ann Emerg Med 2009;53:612-7. https://doi.org/10.1016/j.annemergmed.2008.09.024

8 Cooke M, Fisher J, Dale J, et al. Reducing attendances and waits in emergency departments. A systematic review of present innovations. London: the National Coordinating Centre for the Service Delivery and Organisation, London School of Hygiene and Tropical Medicine 2004.

9 Forero R, McCarthy S, Hillman K. Access block and emergency department overcrowding (http://ccforum.com/ content/15/2/216. Accessed 1 Feb 2013). https://doi. org/10.1186/cc9998

10 Chan SW, NK Cheung, Graham CA, et al. Strategies and solutions to alleviate access block and overcrowding in emergency departments. Hong Kong Med J 2015;21:34552. https://doi.org/10.12809/hkmj144399

11 Dunn R. Reduced access block causes shorter emergency department waiting times: an historical control observational study. Emerg Med 2003;15:232-8. https://doi. org/10.1046/j.1442-2026.2003.00441.x

12 Forero R, Hillman KM, McCarthy S, et al. Access block and ED overcrowding. Emerg Med Australas 2010;22:119-35. https://doi.org/10.1111/j.1742-6723.2010.01270.x 
13 Gilligan P, Winder S, Ramphul N, et al. The referral and complete evaluation time study. Eur J Emerg Med 2010;17:34953. https://doi.org/10.1097/MEJ.0b013e328337b9f1

14 Bullard MJ, Villa-Roel C, Bond K, et al. Tracking emergency department overcrowding in a tertiary care academic institution. Healthc Q 2009;12:99-106. https://doi. org/10.12927/hcq.2013.20884

15 Richardson D. 2. Access block point prevalence survey. The Australasian College for Emergency Medicine, 2008 (https://www.acem.org.au/getattachment/e6442562-06f74629-b7f9-8102236c8b9d/Access-Block-2009-pointprevalence-study.aspx. Accessed 1 Feb 2013).

16 Sun BC, Hsia RY, Weiss RE, et al. Effect of emergency department crowding on outcomes of admitted patients. https://doi.org/10.1016/j.annemergmed.2012.10.026

17 Institute of Medicine Committee on the Future of Emergency Care in the United States Health System. Hospitalbased emergency care: at the breaking point. Washington, DC: National Academies Press 2006 (http://www.nap. edu/catalog/11621.html.Accessed 15 Feb 2013).

18 Wiler JL, Ross MA, Ginde AA. National study of emergency department observation services. Acad Emerg Med 2011;18:959-65. https://doi.org/10.1111/j.15532712.2011.01151.x

19 Gómez-Vaquero C, Soler AS, Pastor AJ, et al. Efficacy of a holding unit to reduce access block and attendance pressure in the emergency department. Emerg Med $\mathrm{J}$ 2009;26:571-2. https://doi.org/10.1136/emj.2008.066076

20 Collins SP, Schauer DP, Gupta A, et al. Cost-effectiveness analysis of ED decision making in patients with non-highrisk heart failure. Am J Emerg Med 2009;27:293-302. https://doi.org/10.1016/j.ajem.2008.02.025

${ }_{21}$ Chun S, Tu JV, Wijeysundera HC, et al. Lifetime analysis of hospitalizations and survival of patients newly admitted with heart failure. Circulation: Heart Failure 2012;5:414-21. https://doi.org/10.1161/CIRCHEARTFAILURE.111.964791

22 Schull MJ, Vermeulen MJ, Stukel TA, et al. Evaluating the effect of clinical decision units on patient flow in seven Canadian emergency departments. Acad Emerg Med 2012;19:828-36. https://doi.org/10.1111/j.15532712.2012.01396.x

${ }_{23}$ Ho EC, Schull MJ, Lee DS. The challenge of heart failure discharge from the emergency department. Curr Heart Fail Rep 2012;9:252-9. https://doi.org/10.1007/s11897-012-0100-1

24 Diercks DB, Peacock WF, Kirk JD, et al. ED patients with heart failure: identification of an observational unit-appropriate cohort. Am J Emerg Med 2006;24:319-24. https:// doi.org/10.1016/j.ajem.2005.11.014

25 Storrow AB, Collins SP, Lyons MS, et al. Emergency department observation of heart failure: preliminary analysis of safety and cost. Congest Heart Fail 2005;11:68-72. https://doi.org/10.1111/j.1527-5299.2005.03844.x

${ }^{26}$ Burkhardt J, Peacock WF, Emerman CL. Predictors of emergency department observation unit outcomes. Acad Emerg Med 2005;12:869-74. https://doi.org/10.1197/j. aem.2005.03.534
27 Venkatesh AK, Geisler BP, Gibson Chambers JJ, et al. Use of observation care in US emergency departments, 2001 to 2008. PLoS One 2011;6:e24326. https://doi. org/10.1371/journal.pone.0024326

28 Peacock WF, Young J, Collins S, et al. Heart failure observation units: optimizing care. Ann Emerg Med 2006;47:2233. https://doi.org/10.1016/j.annemergmed.2005.07.006

29 Fogg C, Griffiths P, Meredith P. Hospital outcomes of older people with cognitive impairment: an integrative review Int J Geriatr Psychiatry 2018;33:1177-97. https://doi. org/10.1002/gps.4919

30 Storrow AB, Collins SP, Lyons MS, et al. Emergency department observation of heart failure: preliminary analysis of safety and cost. Congest Heart Fail 2005;11:68-72. https://doi.org/10.1111/j.1527-5299.2005.03844.x

31 Pal RY, Kuan WS, Koh Y, Death among elderly patients in the emergency department: a needs assessment for endof-life care. Singapore Med J 2017;58:129-33. https://doi. org/10.11622/smedj.2016179

32 Chan GK. Trajectories of approaching death in the emergency department: clinician narratives of patient transitions to the end of life. J Pain Symptom Manage 2011; 42:86481. https://doi.org/10.1016/j.jpainsymman.2011.02.023

${ }_{33}$ Bell D, Ruttenberg MB, Chai E. Care of geriatric patients with advanced illnesses and end-of-life needs in the emergency department. Clin Geriatr Med 2018;34:453-67. https://doi.org/10.1016/j.cger.2018.04.008

${ }^{34}$ Chan GK. End-of-life models and emergency department care. Acad Emerg Med 2004;11:79-86. https://doi. org/10.1197/j.aem.2003.07.019

35 McGrew DM. Chronic illnesses and the end of life. Prim Care 2001;28:339-47. https://doi.org/10.1016/s00954543(05)70026-9

36 Collins SP, Schauer DP, Gupta A, et al. Cost-effectiveness analysis of ED decision making in patients with non-highrisk heart failure. Am J Emerg Med 2009;27:293-302. This is an important cost-effectiveness analysis of three possible ED disposition strategies for acute HF: a) discharge home; b) observation unit, or c) inpatient admission. As the baseline rate of events increased, the cost-effectiveness of observation units decreased and hospitalization became the more effective strategy. https://doi.org/10.1016/j. ajem.2008.02.025

37 Savioli G, Ceresa IF, Macedonio S, Trauma Coagulopathy and its outcomes. Medicina 2020;56:205. https://doi. org/10.3390/medicina56040205

38 Iserson KV, Moskop JC. Triage in medicine, part I: concept, history, and types. Ann Emerg Med 2007;49:275-81.

39 Hamamoto J, Yamase H, Yamase Y. Impacts of the introduction of a triage system in Japan: a time series study. Int Emerg Nurs 2014;22:153-8.

40 Gerdtz MF, Collins M, Chu M, et al. Optimizing triage consistency in Australian emergency departments: the Emergency Triage Education Kit. Emerg Med Australas 2008;20:250-9. https://doi.org/10.1111/j.17426723.2008.01089.x 
41 FitzGerald G, Jelinek GA, Scott D, et al. Emergency department triage revisited. Emerg Med J 2010;27:86-92. https://doi.org/10.1136/emj.2009.077081.

42 Bullard MJ, Musgrave E, Warren D, et al. Revisions to the Canadian Emergency Department Triage and Acuity Scale (CTAS) Guidelines 2016. CJEM 2017;19:S18-27. https:// doi.org/10.1017/cem.2017.365

43 Wuerz RC, Milne LW, Eitel DR, et al. Reliability and validity of a new five-level triage instrument. Acad Emerg Med 2000;7:236-42. https://doi. org/10.1111/j.1553-2712.2000.tb01066.x

44 Christ M, Grossmann F, Winter D, et al. Modern triage in the emergency department. Dtsch Arztebl Int 2010;107:8928. https://doi.org/10.3238/arztebl.2010.0892

45 FitzGerald G, Jelinek GA, Scott D, et al. Emergency department triage revisited. Emerg Med J 2010;27:86-92. https://doi.org/10.1136/emj.2009.077081

46 Alquraini M, Awad E, Hijazi R. Reliability of Canadian Emergency Department Triage and Acuity Scale (CTAS) in Saudi Arabia. Int J Emerg Med 2015;8:80. https://doi. org/10.1186/s12245-015-0080-5
47 Considine J, Lucas E, Payne R, et al. Analysis of three advanced practice roles in emergency nursing. Australas Emerg Nurs J 2012;15:219-28. https://doi.org/10.1016/j.aenj.2012.10.001

48 Hodge A, Hugman A, Varndell W, et al. A review of the quality assurance processes for the Australasian Triage Scale (ATS) and implications for future practice. Australas Emerg Nurs J 2013;16:21-9. https://doi.org/10.1016/j. aenj.2012.12.003

49 Fitzgerald GJ, Robertson CE, Little K, et al. The urgency distribution of an accident and emergency department's workload. Arch Emerg Med 1986;3:225-30. https://doi. org/10.1136/emj.3.4.225

50 ACCORDO 25/10/2001 Accordo tra il Ministero della Salute, le regioni e le province autonome sul documento di Linee-guida sul sistema di Emergenza sanitaria Concernente: triage intraospedaliero (valutazione gravità all'ingresso) e chirurgia della mano e microchirurgia nel sistema dell'emergenza-urgenza sanitaria (https://www. gazzettaufficiale.it/eli/id/2001/12/07/01A12203/sg; http:// www.salute.gov.it/imgs/C_17_normativa_1747_allegato. pdf). 